\title{
COVID-19 and the neurological disease therapeutic pipeline
}

\author{
Gregory A. Jicha ${ }^{\circledR}$ and Erin L. Abner
}

\section{From the interruption of clinical trials by shelter-in-place orders to the challenges involved in safely collecting biofluid samples, drug development for neurological disease was hit hard by the COVID-19 pandemic this year. However, the field has responded with innovative solutions, and 2021 could see the therapeutic pipeline flowing again.}

Severe acute respiratory syndrome coronavirus 2 (SARS-CoV-2), which emerged in late 2019 and rapidly spread across the globe, led to an almost complete worldwide shutdown of research targeting neurological diseases by mid-March ${ }^{1-7}$. Thus, the therapeutic pipeline for neurological diseases - which had previously been rapidly expanding - came to a grinding halt. Public health measures necessary to control the spread of coronavirus disease 2019 (COVID-19), including social distancing and shelter-in-place orders, have impacted every stage of therapeutic development, from basic science discovery to the manufacturing and distribution of both approved and experimental neurological therapeutics. However, the impact of COVID-19 has been most profound in the area of active clinical trial conduct, where both the medical research workforce and clinical trial participants were substantially limited in their ability to meet the rigorous demands and tight time schedules inherent in human clinical trial activities ${ }^{1,2}$.

Many experimental therapeutics require dosing via on-site administration, as well as carefully timed outcome measure assessments; thus, the interruption of clinical trials by the COVID-19 pandemic has severely damaged the implementation of the precise protocols required to obtain proof of safety and efficacy ${ }^{1,2}$. For example, an article by Joseph Broderick and colleagues ${ }^{1}$ published in June described the impact of COVID-19 on the NIH StrokeNet, a network of centres performing clinical trials in stroke. An initial complete shutdown of all trials across all centres was coordinated centrally and was followed by a phased research restart plan that took into account local situations and regulatory oversight. This approach was successful in re-engaging research activities to at least some degree within 55 days in all but one of the ongoing studies. However, the success of the restart activities and the long-term effects of the shutdown are not yet known.
The interruption of essential components of clinical trial conduct that are necessary for advancement to the next stage of therapeutic development (and/or eventual approval of the experimental therapeutics) has created challenges for both sponsors and regulatory agencies, who have been left uncertain as to how to handle these unprecedented, widespread disruptions $\mathrm{s}^{2,3}$. Nevertheless, solutions have been proposed and have started being implemented. Remote assessments to allow distanced trial conduct are under rapid development and include the use of telephone evaluations, video conference-assisted evaluations and other remote digital strategies ${ }^{1,6}$. Indeed, Broderick et al. ${ }^{1}$ recommended that trial sites incorporate remote assessments as

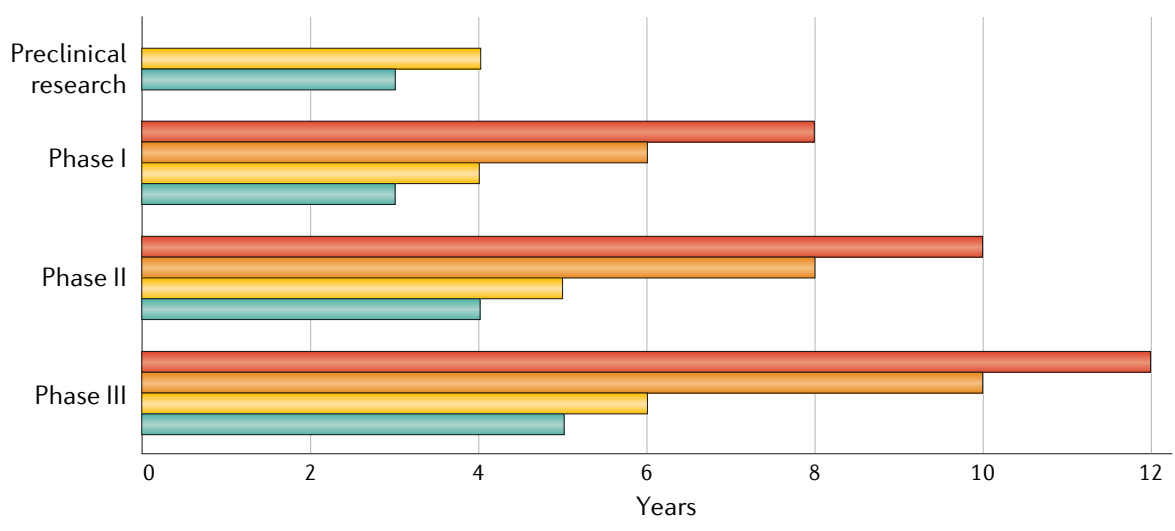

COVID-19 impact if trial engagement is cut by $50 \%$ in the future COVID-19 impact if trial has to be redone COVID-19 direct impact

Traditional timeline

Fig. 1 Potential delays in the pipeline for neurological therapeutic development resulting from the COVID-19 pandemic. Time required for each stage of clinical therapeutic development and the potential impact of COVID-19 on the developmental timeline based on the observed effects of the pandemic so far. Direct COVID-19 impact is a reality, and will extend the timeline for preclinical research and all phases of subsequent development for ongoing trials by a year or more, depending on the duration of the pandemic. If delays, potential need for repetition of studies and reduced likelihood of research engagement remain problematic over the longer term, an average 15-year development programme could double to more than 30 years for drugs that are currently in the early preclinical phase. 


\section{Key advances \\ - Understanding the impact of COVID-19 \\ on diverse aspects of clinical trials ${ }^{1}$. \\ - Development of statistical approaches to mitigate the impact of missing data ${ }^{3}$. \\ - Safely managing biospecimen collections that are essential for establishment of disease modification ${ }^{4}$. \\ - Overcoming site-specific barriers to safe clinical trial conduct ${ }^{2}$. \\ - Maintaining a sufficiently large and diverse pool of research participants ${ }^{5}$.}

Suzanne Schindler and colleagues published a set of recommendations ${ }^{4}$ for the collection and analysis of biofluids in Alzheimer disease research during the COVID-19 pandemic. These recommendations include adherence to universal precautions and biosafety level-1 protocols for most laboratory procedures that do not generate aerosols; use of additional safety measures if procedures have the potential to generate aerosols; clear identification of biosamples collected from individuals with known SARS-CoV-2 infection, as well as provision of this information to anyone receiving such samples; and handling all samples collected during the pandemic as if they contain SARS-CoV-2, given the high rate of asymptomatic infection.

\section{4 the innovation spurred by this crisis will move the field forward in new ways}

The COVID-19 pandemic has also had a downstream effect on the funding that allows the therapeutic pipeline to remain solvent. Maintaining a workforce skilled in clinical trial conduct has been a challenge with widespread furloughs, layoffs and economic collapse threatening not only current but also future clinical trial conduct ${ }^{2}$. In addition, the need for additional personal protective equipment, COVID-19 testing of research participants and staff, implementation of new equipment and training to facilitate biomarker collection, and the introduction of remote assessments have all contributed to an increase in the financial costs involved in running clinical trials. In an article ${ }^{2}$ published in September this year, we made several recommendations for overcoming site-specific barriers to clinical trial conduct during the COVID-19 era, including the allocation of funds to standing site support with the aim of maintaining a research-ready infrastructure for future trials.

Most importantly, COVID-19 has, understandably, negatively affected the interest and willingness of many people to engage in experimental clinical trials ${ }^{2,5,7}$ (FIG. 1). In another key publication from 2020, Daniel Lackland and colleagues ${ }^{5}$ highlighted the potential for the COVID-19 pandemic to further limit the participation of historically underrepresented groups in clinical trials and recommended that the field increases efforts to recruit participants from these groups. Managing safety concerns and overcoming a growing reluctance to engage in research at this time will require innovative solutions, and implementing these solutions will require widespread efforts to educate potential research volunteers on risk reduction measures. Potential volunteers will also need to be educated on the importance of continuing to move therapeutic research forward in order to advance the development of new medicines for the treatment of neurological diseases. Without such efforts, the impact of the COVID-19 pandemic on the therapeutic pipeline for neurological diseases could extend indefinitely.

It remains uncertain as to whether effective solutions to the problems induced by the pandemic can be developed, or whether the many affected trials will be left with inconclusive results, necessitating repeat studies that would cost additional years and millions of US dollars (FIG. 1). In addition, studies scheduled to start this year have been delayed, closing the valve on our previously robust pipeline of new experimental therapeutics even more tightly. Clearly, the impact of COVID-19 on research sites responsible for the conduct of human clinical trials has been profound ${ }^{1,2}$. Nevertheless, the innovation spurred by this crisis will move the field forward in new ways, ensuring that 2021 will be a year that allows the therapeutic pipeline to resume its pre-pandemic vigorous flow. Researchers and clinicians have formed alliances to address barriers to research advancement, allowing new research into statistical techniques to handle the current disruptions to clinical trial conduct ${ }^{3}$, enabling the production of guidelines for enhanced safety of research conduct ${ }^{1,4}$, and spurring on the development and validation of novel remote assessment techniques ${ }^{1,6}$.

Gregory A. Jicha (iD ${ }^{1,2 \otimes}$ and Erin L. Abner ${ }^{2,3}$ 'Department of Neurology, University of Kentucky College of Medicine, Lexington, $K Y, U S$.

${ }^{2}$ The Sanders-Brown Center on Aging, University of Kentucky College of Medicine, Lexington, KY, US.

${ }^{3}$ Department of Epidemiology, University of Kentucky College of Public Health, Lexington, KY, US.

凶e-mail:gajich2@email.uky.edu

https://doi.org/10.1038/s41582-020-00445-w

1. Broderick, J. P. et al. National Institutes of Health StrokeNet during the time of COVID-19 and beyond. Stroke 51, 2580-2586 (2020).

2. Rhodus, E. K., Bardach, S. H., Abner, E. L., Gibson, A $\&$ Jicha, G. A. COVID-19 and geriatric clinical trials research. Aging Clin. Exp. Res. 32, 2169-2172 (2020).

3. Cro, S., Morris, T. P., Kahan, B. C., Cornelius, V. R. $\&$ Carpenter, J. R. A four-step strategy for handling missing outcome data in randomised trials affected by a pandemic. BMC Med. Res. Methodol. 20, 208 (2020).

4. Schindler, S. E. et al. Maximizing safety in the conduct of alzheimer's disease fluid biomarker research in the era of COVID-19. J. Alzheimers Dis. 76, 27-31 (2020).

5. Lackland, D. T., Sims-Robinson, C., Jones Buie, J. N. $\&$ Voeks, J. H. Impact of COVID-19 on clinical research and inclusion of diverse populations. Ethn. Dis. 30 , 429-432 (2020).

6. Andrews, J. A. et al. Amyotrophic lateral sclerosis care and research in the United States during the COVID-19 pandemic: Challenges and opportunities. Muscle Nerve 62, 182-186 (2020).

7. Bardach, S. H., Gibson, A. K., Rhodus, E. K. \& Jicha, G. A. Will "social distancing" lead to future "research distancing": A reflection on COVID-19 impacts on Alzheimer's disease research. Alzheimers Dement. 6, e12045 (2020).

\section{Competing interests}

G.A.J. has received contract research funding from AbbVie, Biohaven, Eisai, Lilly, Neurovision, Novartis and Suven. E.L.A. declares no competing interests. 